\title{
Preliminary Investigations on the Applicability of the Fixed Point Transformations-Based Adaptive Control for Time-Delayed Systems
}

\author{
Bertalan Csanádi ${ }^{\dagger}$, Tamás Haidegger ${ }^{\star, \ddagger}$, Hemza Redjimi $^{\ddagger}$, and József K. Tar ${ }^{\star, \ddagger}$ \\ ${ }^{\dagger}$ Doctoral School of Applied Informatics and Applied Mathematics, \\ ${ }^{\star}$ University Research and Innovation Center, Antal Bejczy Center for Intelligent Robotics (ABC iRob), \\ $\$$ John von Neumann Faculty of Informatics, \\ Óbuda University, Budapest, Hungary \\ \{csanadi.bertalan@phd., tar.jozsef@nik.\}uni-obuda.hu, haidegger@irob.uni-obuda.hu, hamza-redjimi@hotmail.fr
}

\begin{abstract}
In this paper, for the first time, a possible tackling of the problem of known time-delay by the use of a Fixed Point Transformation-based adaptive controller is investigated. This approach at first transform the control task into a fixed point problem then solves it via iteration. The preliminary results that were obtained by numerical simulations for a strongly nonlinear controlled system, a van der Pol oscillator, are promising. It is expedient to make further, systematic investigations.

Index Terms-Adaptive Control, Fixed Point TransformationsBased Adaptive Control, Robust Fixed Point Transformation, Time-Delayed Systems.
\end{abstract}

\section{INTRODUCTION}

In the field of Model Predictive Control (MPC), typical practical problems used to arise as:

1) the imprecision and incompleteness of the available system models;

2) the lack of possibility for measuring each state variable;

3) in many cases, the state variables depend on the directly measurable quantities through uncertain parameters that, show great variability;

4) the high complexity, often too high order of the available models;

5) limitations in the control actions;

6) in many cases, we have strongly underactuated systems, that means that it is impossible to precisely control each state variable simultaneously;

7) in many cases, between the observations and the control action some time-delay is present; depending on the circumstances the extent of the delay can be known in advance as a fixed value or it may be uncertain.

The problem groups 1, 2 and 6 are typical in modeling biological processes: various models are developed for describing the same phenomenon. For example, for the description of the human glucose-insulin system Bergman's "Minimal Model" has only a few state variables [1], [2] while the more complex model by Dalla Man et al. [3] works with 10 compartments. In practice, only the glucose concentration in the blood can be measured, and no information is obtained on the other state variables. For the control of the numerous state variables, there is only one control signal, the subcutaneous insulin injection rate. In certain cases, e.g. in the anaesthesia models "fictitious" state variables are applied in the models that do not cover real parts of the human body. They role simply is to describe certain time-delay effects (e.g., [4], [5]). In certain systems for which typical regimes of operation can be identified the idea of "situational control" cab be also applied (e.g., [6], [7]).

The occurrence of problem group 3 is typical in the field of anaesthesia: the sensitivity of the patients are related to the measurable signals (either the "Bispectral Index" [8] or the EEG signals of the central nervous system processed by wavelet analysis [9]), and this relation is described by the strongly nonlinear function of parameters that have great interpatient deviations.

The presence of problem group 5 is typical in chemical reactions and biological systems, too. It is possible to produce arbitrary selective ingress of certain reagents, but we cannot selectively extract them from a continuously stirred tank reactors or the living organisms. In this case the controller has to stop at the minimal possible (i.e. 0) ingress rate and has to wait until the appropriate concentration decreases by natural processes [10], [11]. It also worths noting that the introduction of even a pure reagent dilutes the others that corresponds to a type of input coupling [12].

For the example of group 4, the dynamically coupled systems of many degree of freedom can be mentioned. Due to such couplings the order of the time-derivatives that appear in the equations of motion are impractically high and cannot be handled in an efficient manner. In such cases for the design of the controller intentionally distorted, reduced order models are used. In the realm of "Linear Time-Invariant" (LTI) systems the "Model Reduction Techniques" are based on the use of the Krylov bases (e.g. [13], [14]). In 1950 C. Lanczos [15] and in 1951 W.E. Arnoldi [16] developed transformations that produced vectors of the elements of the Krylov basis to obtain an approximate model that more appropriate for control purposes. In the frequency domain an alternative approach was the use of the Padé approximants [17]: the basic idea is to produce a truncated Taylor series expansion of the transfer function in the frequency range of practical interest, and instead using 
this polynomial that does not behave well in the infinity a fractional expression having the same truncated Taylor series is applied [18]. This fractional expression behaves "well" in the frequency domains of not primary interest. An alternative model reduction technique is the application of fractional order derivatives (e.g., [19], [20]) instead of integer order ones. In this manner, these fractional derivatives have long term memory and can be described only by a few parameters [21]. The method came to fashion in the thirties of the 20th century, for describing viscoelastic phenomena [22], [23], and nowadays it is popular in active car suspension systems (e.g. [24], [25]). This problem group was successfully addressed by the fixed point transformation-based adaptive controller for stable nonlinear systems in [26].

The problem groups 1-6 were already successfully addressed by the "Fixed Point Transformation-Based Adaptive Control Design Method" reported in [27]. However, the problem group 7, i.e., the time-delay problems were not systematically investigated from this point of view though the suggested method is essentially based on the application of time-delays. The relevant scientific antecedents are briefly summarized in the sequel.

\section{A. Fixed Point Transformation-Based Adaptive Control Design}

The fixed point transformation-based adaptive control method appeared as a "competitor" of the prevailing design technique for strongly nonlinear systems, i.e., "Lyapunov's Direct Method” . In 1892 Lyapunov defended his PhD Thesis, in which he investigated the stability of motion of nonlinear systems [28]. Realizing that in the case of the great majority of the real problems, the equations of motion cannot be obtained in closed analytical form, therefore it is impossible to investigate the stability by studying the behavior of the unknown solutions, he introduced various stability definitions, and an estimation technique that made it possible to determine the stability without knowing other fine details of the motion. In the sixties of the past century, his work was translated to English [29], and by the nineties it appeared as the main design tool for adaptive controllers (e.g., [30], [31]), Model Reference Adaptive Controllers (MRAC) [32], [33], [34]. In spite of its great virtues, this method has some drawbacks such as mathematical complexity, requiring satisfactory conditions that frequently restrict much more than the necessary and satisfactory conditions, allowing the use of a great number of arbitrary control parameters that may be optimized by evolutionary methods (e.g., [35], [36]). A fresh and emerging modeling approach is the Tensor Product Model (TP) (e.g., [37], [38]) in which the Lyapunov function technique can be combined with Linear Matrix Inequalities for controller design purposes. It was recently studied in connection with the automatic treatment of Diabetes Mellitus in [39].

The main idea of the fixed point transformation-based approach, i.e. that a given technical task can be transformed into a fixed point problem that subsequently can be solved via iteration goes back to the $17^{\text {th }}$ century. The well known root finding algorithm invented by Newton and Raphson obtains considerable attention even in our days [40], [41], [42]. In 1922, Banach introduced contractive mappings in linear, normed, complete metric spaces. Due to his idea, the application of the original idea was extended to quite abstract spaces [43].

Problem groups 1, 2, and 3 were recently addressed e.g. in [44], [45]. Regarding problem group 4 (model reduction) a quite simple and novel approach was presented in [46]. By the use of a special polynomial numerical differentiator problem classes of relatively high order can be tackled by this method [47]. For the limitations or the control signals (class 5) [48], [49], [12] can be mentioned. For problem group 6 a novel approach aiming at the combination of the adaptive and optimal controllers preliminary results were published in [50]. It worths noting that the method was successfully combined with fractional order systems [51], too.

It is important to note that it was found possible to combine the fixed point transformation-based method with the traditional adaptive controllers on the basis of a simple, geometric interpretation (e.g., [52], [53]).

In the present paper, problem group 7 is tackled. The relevant literature is briefed in the next subsection.

\section{B. The Prevailing Solutions According to the Current Litera- ture}

On the basis of a rough and preliminary literature overview it can be stated that the Linear Systems [54], [55], [56], and within this class the Positive Linear Systems [57] form a particular problem class that was well tackled on the basis of the generalization/extension of Lyapunov's method (namely the use of the Lyapunov-Krasovskii functional) [58], [59]. An important class is in which the time-delay itself is not constant [60]. Other approaches connect they results to the concept of "robust stability" [61], [62], [55], [63].

\section{Dealing Time Delay in Fixed Point TRANSFORMATION-BASED AdAPTIVE CONTROL}

For the success of the controller the purely kinetically prescribed tracking error relaxation may have great significance especially when the order of the controller is 2. Utilizing the fact that $\forall \Lambda=$ const. $>0$ parameter the function $x(t)=e^{-\Lambda\left(t-t_{0}\right)} x\left(t_{0}\right)$ exponentially converging to zero satisfies the ordinary differential equation $\left(\frac{\mathrm{d}}{\mathrm{d} t}+\Lambda\right) x(t)=0$, the following plausible variants can be investigated for kinetically prescribed error-relaxation: a PD-type tracking error $e(t) \stackrel{\text { def }}{=}$ $q^{N}(t)-q(t)$ relaxation can be obtained from the prescription $\left(\Lambda+\frac{\mathrm{d}}{\mathrm{d} t}\right)^{2} e(t) \equiv \mathrm{o}$ leading to

$$
\ddot{q}^{D e s}=\ddot{q}^{N}+2 \Lambda \dot{e}+\Lambda^{2} e .
$$

A PID-type error-relaxation can be obtained from the integrated error $e_{\text {int }}(t) \stackrel{\text { def }}{=} \int_{t_{0}}^{t} e(\xi) \mathrm{d} \xi$ requiring $\left(\Lambda+\frac{\mathrm{d}}{\mathrm{d} t}\right)^{3} e_{\text {int }}(t) \equiv$ o that leads to

$$
\ddot{q}^{D e s}=\ddot{q}^{N}+3 \Lambda \dot{e}+3 \Lambda^{2} e+\Lambda^{3} e_{i n t} .
$$




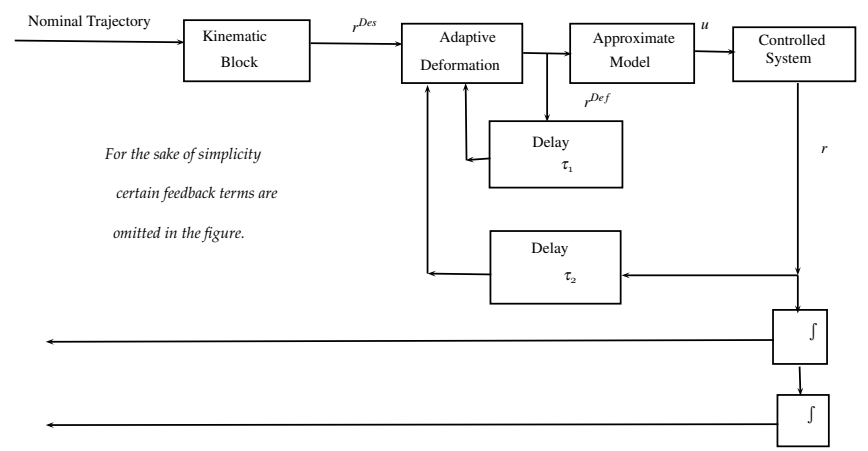

Fig. 1. The structure of the Fixed Point Transformation-Based Adaptive Controller

Further plausible option would be the use of the error relaxation strategy of the Variable Structure / Sliding Mode Robust Controllers (VS/SM) (e.g. [64], [65]) in which, due to the lack of the precise dynamic model, no attempt is done for the precise realization of the above PD or PID-type strategy. Instead, a so called Error Metrics is introduced that may or may not contain integrated error: $S_{P D}(t) \stackrel{\text { def }}{=}\left(\Lambda+\frac{\mathrm{d}}{\mathrm{d} t}\right)\left(q^{N}(t)-q(t)\right)$ or $S_{P I D}(t) \stackrel{\text { def }}{=}\left(\Lambda+\frac{\mathrm{d}}{\mathrm{d} t}\right)^{2} \int_{t_{0}}^{t}\left(q^{N}(\xi)-q(\xi)\right) \mathrm{d} \xi$, and the controller tries to so prescribe $\dot{S}$ that it will be driven zero during finite time or exponentially as in [66] without minding the precise realization of this prescription. As soon as $S$ is kept at zero similar exponential-type error relaxation is initiated as in the case of the PD or PID controls.

In Fig. 1, at first, $\ddot{q}^{D e s}$ is produced by the "Kinematic Block", following that it is adaptively deformed by the block of "Adaptive Deformation", and this deformed signal is used for the calculation of the control force $u$ that exerted on the "Controlled System" causing the "Realized Response" $r \equiv \ddot{q}$. Due to the combination of the approximate system model and the observed response in [27], the concept of the "response function" $r=f\left(r^{D e f}\right)$ was introduced that for its input provides the realized response. The deformation was realized by the "Robust Fixed Point Transformation" for single variable $f: \mathbb{R} \mapsto \mathbb{R}$ in the case of an analog controller as

$$
\begin{gathered}
r^{D e f}(t+\delta t) \stackrel{\text { def }}{=} G\left(r^{D e f}(t), \ddot{q}^{D e s}(t+\delta t)\right) \equiv\left(r^{D e f}(t)+K_{c}\right) \times \\
{\left[1+B_{c} \tanh \left(A_{c}\left(f\left(r^{D e f}(t)\right)-\ddot{q}^{D e s}(t+\delta t)\right)\right)\right]-K_{c}}
\end{gathered}
$$

in which $\ddot{q}^{D e s}$ is made of the integrated tracking error, the error, and the time-derivative of the error, therefore it varies quite slowly with respect to the instantaneously variable $\ddot{q}$ that immediately can be set by an appropriate control force $u$. With the approximation of $\ddot{q}^{\text {Des }}=$ const. the solution of the control task, i.e. the deformed response $r_{\star}$ for which $f\left(r_{\star}\right)=r^{\text {Des }}$ evidently is the fixed point of the function defined in Eq. (3). In the case of a digital controller $\delta t$ can be equal to its cycle time. According to Banach's Fixed Point Theorem [43] the sequence generated by the function $G$ converges to $r_{\star}$ if this function is contractive. For achieving contractivity in the vicinity of $r_{\star}$ it is not difficult to set the three control parameters $K_{c}, B_{c}$, and $A_{c}$ [27]. In Fig. 1 this original setting corresponds to $\tau_{1}=\tau_{2}=\delta t$ that corresponds to the assumption that the effect of $u$ immediately becomes observable, and in the given control cycle, the controller "learns" from the results of the previous one. For achieving good result, it is necessary to set $\delta t$ small enough to well track the variation of the of the nominal motion and the dynamics of the controlled system. With other words, the information that was gathered by studying the system's behavior in the previous control cycle must not be too "obsolete" in the given time.

The flexibility of the arrangement in Fig. 1 consist in the fact that $\tau_{1}$ may be different to $\tau_{2}$, therefore if it is assumed e.g. that the suggested control force will be exerted on the controlled system with the delay $\tau$, and the observed response also "travels" to the controller during the same time, a reasonable setting for the adaptation will be $\tau_{1}=\delta t+2 \tau$, and $\tau_{2}=\delta t$. In the sequel the effects of such a priori known time delays will be studied via numerical simulations.

\section{Simulation InVESTigations}

In order to obtain rich dynamic behavior, a strongly nonlinear modification of the original van der Pol oscillator was suggested with the equation of motion as follows:

$$
\begin{gathered}
\ddot{q}=-k_{1} q-k_{2} \tanh \left(\frac{q^{3}}{a^{3}}\right)-b\left(q^{2}-c^{2}\right) \dot{q} \\
-d \tanh \left(\frac{q}{e}\right) \dot{q}^{2}+F u,
\end{gathered}
$$

in which $k_{1}$ corresponds to a spring constant, $k_{2}$ and $a$ are related to a limited strengthening of the spring constant, $b$ and $c$ are responsible for the instability of the equilibrium point of the free system at $q=0, \dot{q}=0$, and for bringing about nonlinear oscillations of the system, while $d$, and $e$ are responsible for a strongly nonlinear damping that used to be caused by turbulences if the controlled systems moves in air or some liquid, and $F$ determines the effectivity of the control force $u[N]$. In the simulations three parameter sets were used: a) the nominal trajectory was generated by the free motion of an oscillator with the initial state nearby of the unstable equilibrium point, b) the oscillator with approximate model parameters used for the generation of the control forces, and c) the controlled system's exact parameters as they are given in Table II. The control parameters are provided by Table II.

TABLE I

THE MODEL PARAMETERS

\begin{tabular}{|l|c|c|c|}
\hline Parameter & Trajectory Generator & Approximate & Exact \\
\hline$k_{1}\left[\frac{1}{s^{2}}\right]$ & 8 & 9 & 8 \\
\hline$k_{2}\left[\frac{m}{s^{2}}\right]$ & 2 & 1.5 & 2 \\
\hline$a[m]$ & 2 & 3 & 2 \\
\hline$b\left[\frac{1}{m^{2} s}\right]$ & 3 & 4 & 3 \\
\hline$c[m]$ & 3 & 0.44 & 0.3 \\
\hline$d\left[\frac{m}{s^{2}}\right]$ & 2 & 3.2 & 2 \\
\hline$e[m]$ & 1 & 3 & 1 \\
\hline$F\left[\frac{m}{N s^{2}}\right]$ & 0 & 4 & 1 \\
\hline
\end{tabular}


TABLE II

THE CONTROL PARAMETERS

\begin{tabular}{|c|c|}
\hline Parameter & Value \\
\hline$\Lambda\left[\frac{1}{s}\right]$ & 6 \\
\hline$B_{c}[$ nondimensional $]$ & -1 \\
\hline$K_{c}\left[\frac{m}{s^{2}}\right]$ & $5 \times 10^{7}$ \\
\hline$A_{c}\left[\frac{s^{2}}{m}\right]$ & $\frac{1}{10\left|K_{c}\right|}$ \\
\hline$\delta t[s]$ & $10^{-4}$ \\
\hline
\end{tabular}

In the simulations, (2) was applied with the modification that allowed a little imprecision in the calculation of the tracking error, as $e(t) \stackrel{\text { def }}{=} q^{N}(t)-q(t-\tau)$ together with its consequences in the feedback terms. The simulations were made by INRIA's SCILAB simulator version 5.5.2 and its appropriate XCOS graphical programmer with the numerical integrator "Sundials/CVODE-ADAMS-FUNCTIONAL" with the standard precision settings and an automatically chosen maximal step length in the integration.

In the first set of simulations a quite negligible delay time $\tau=10^{-10}[s]$ was chosen in the case of a non-adaptive control. The trajectory tracking is revealed by Fig. 2. As the graphs of the phase trajectories also reinforces it (Fig. 3), the generator of the nominal trajectory produced strongly nonlinear oscillations.
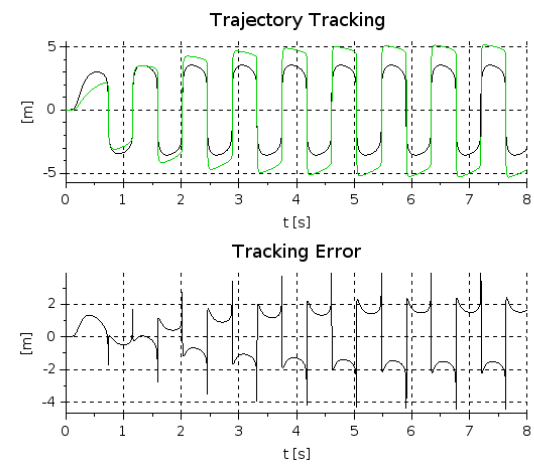

Fig. 2. The trajectory tracking and tracking error of the non-adaptive controller for $\tau=10^{-10}[s]$ (nominal trajectory: black, simulated trajectory: green lines

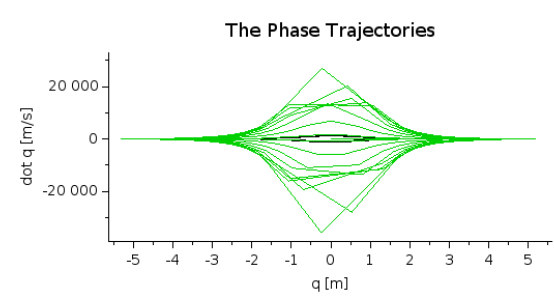

Fig. 3. The phase trajectories of the non-adaptive controller for $\tau=10^{-10}[s]$ (nominal trajectory: black, simulated trajectory: green lines

The adaptive counterpart of Figs. 2 and 3 are displayed in Figs. 4 and 5. Figure 6 well reveals the significance of the adaptive deformation of the controller.

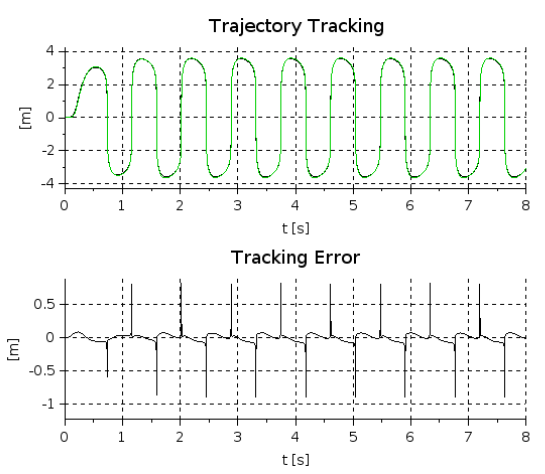

Fig. 4. The trajectory tracking and tracking error of the adaptive controller for $\tau=10^{-10}[s]$ (nominal trajectory: black, simulated trajectory: green lines

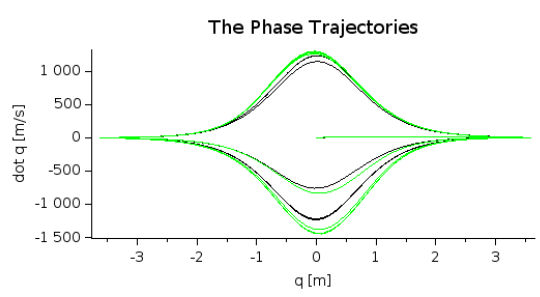

Fig. 5. The phase trajectories of the adaptive controller for $\tau=10^{-10}[s]$ (nominal trajectory: black, simulated trajectory: green lines

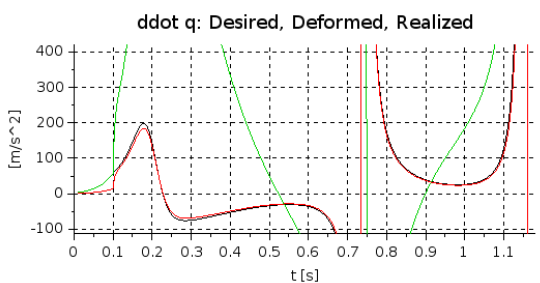

Fig. 6. The "Desired" (black line), the adaptively "Deformed" (green line), and the "Realized" (red line) $\ddot{q}$ values versus time for the adaptive controller for $\tau=10^{-10}[s]$ (a zoomed in excerpt)

In the second set of simulations, the setting $\tau=4 \times 10^{-4}[s]$ was applied. According to Fig. 7 the non-adaptive controller collapsed but the adaptive one still worked well (Fig. 8). In Fig. 9, typical chattering, characteristic to leaving the convergence zone of the RFPT-based adaptive controllers, appears in $\ddot{q}^{D e f}$ that already was investigated, e.g., in [67].

\section{CONCLUSIONS}

On the basis of the simulation results, it can be definitely stated, that the RFPT-based adaptive controller can reduce the simultaneous effects of the modeling errors and the delayed observation caused "obsolescence" of the information used in the learning process, as far as the precision of trajectory tracking is concerned. The allowable delay in the observations strongly depends on the dynamic properties of the nominal trajectory to be tracked. The sharp jumps in the nominal trajectory of the van der Pol Oscillator serve as hard and critical examples. It is expected that for "smoother" systems longer delays can be allowed. Our further aim is to make simulation investigations with randomly varying time delays by adding 


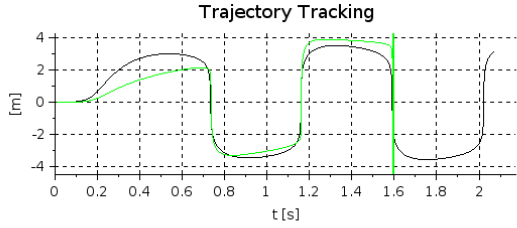

Tracking Error

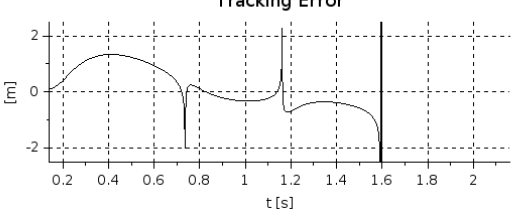

Fig. 7. The trajectory tracking and tracking error of the non-adaptive controller for $\tau=4 \times 10^{-10}[s]$ (nominal trajectory: black, simulated trajectory: green lines)

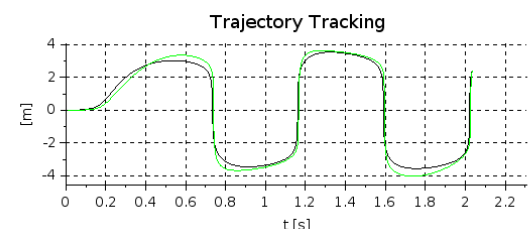

$\mathrm{t}[\mathrm{s}]$

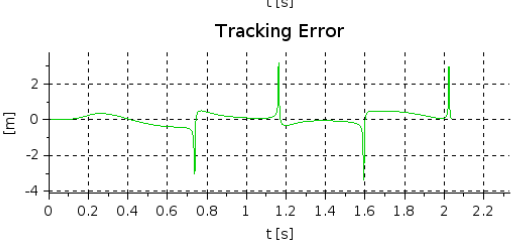

Fig. 8. The trajectory tracking and tracking error of the adaptive controller for $\tau=4 \times 10^{-10}[s]$ (nominal trajectory: black, simulated trajectory: green lines)

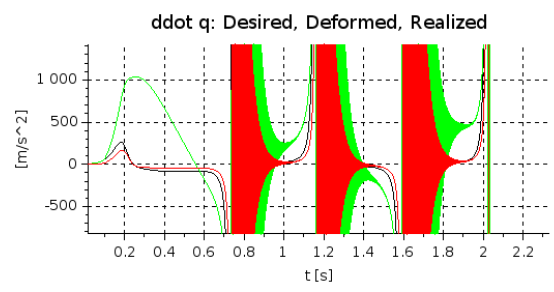

Fig. 9. The "Desired" (black line), the adaptively "Deformed" (green line), and the "Realized" (red line) $\ddot{q}$ values versus time for the adaptive controller for $\tau=4 \times 10^{-10}[s]$ (a zoomed in excerpt)

time-stamps to the delayed signals to check the operation of the adaptive control method in information networks in which the signal packets can travel through various nodes of the network.

\section{ACKNOWLEDGMENT}

The research was supported by the Doctoral School of Applied Informatics and Applied Mathematics of Óbuda University.

\section{REFERENCES}

[1] R. Bergman, Y. Ider, C. Bowden, and C. Cobelli, "Quantitative estimation of insulin sensitivity," Am. J. Physiol. Endocrinol. Metab., vol. 236, pp. E667-E677, 1979.

[2] C. Juhász, Medical Application of Adaptive Control, Supporting InsulinTherapy in case of Diabetes Mellitus (PhD thesis). Budapest University of Technology and Economics, Budapest, Hungary, 1997.
[3] C. D. Man, R. Rizza, and C. Cobelli, "Meal simulation model of glucoseinsulin system," IEEE TRANSACTIONS ON BIOMEDICAL ENGINEER$I N G$, vol. 54, no. 10, pp. 1740-1749, 2007.

[4] C. Ionescu, R. De Keyser, B. Torrico, T. Smet, M. Struys, and J. NormeyRico, "Robust predictive control strategy applied for Propofol dosing using BIS as a controlled variable during anesthesia," IEEE Transactions on Biomedical Engineering, vol. 55, no. 9, pp. 2161-2170, 2008.

[5] I. Naşcu, R. Oberdieck, and E. Pistikopoulos, "Offset-free explicit hybrid model predictive control of intravenous anaesthesia," In: Proc. of the of the 2015 IEEE International Conference on Systems, Man, and Cybernetics, October 9-13, 2015, Hong Kong, pp. 2475-2480, 2015.

[6] L. Madarász, R. Andoga, L. Főzô, and T. Lázár, Situational control modeling and diagnostics of large scale systems, In I. Rudas, J. Fodor, J. Kacprzyk (eds.) Towards Intelligent Engineering and Information Technology pp. 153-164. Springer Verlag, Heidelberg, 2009.

[7] R. Andoga, L. Főző, L. Madarász, J. Považan, and J. Judičák, "Basic approaches in adaptive control system design for small turbo-compressor engines," In: Proc. of the IEEE 18th International Conference on Intelligent Engineering Systems (INES 2014), from 3 to 5 July, 2014, Tihany, Hungary, pp. 95-99, 2014.

[8] M. Struys, H. Vereecke, A. Moerman, E. Jensen, D. Verhaegen, N. De Neve, F. Dumortier, and E. Mortier, "Ability of the Bispectral Index, autoregressive modelling with exogenous input-derived auditory evoked potentials, and predicted Propofol concentrations to measure patient responsiveness during anesthesia with Propofol and Remifentanil," Anesthesiology, vol. 99, no. 4, pp. 802-812, 2003.

[9] T. Zikov, S. Bibian, G. Dumont, M. Huzmezan, and C. Ries, "Quantifying cortical activity during general anesthesia using wavelet analysis," IEEE Transactions on Biomedical Engineering, vol. 53, no. 4, pp. 617632, 2006.

[10] B. Bequette, "Non-linear control of chemical processes: A review," Ind. Engng. Chem. Res., vol. 30, pp. 1391-1413, 1991.

[11] N. Moldoványi, Model Predictive Control of Crystallisers. PhD Thesis, Department of Process Engineering, University of Pannonia, Veszprém, Hungary, 2012.

[12] J. Tar, I. Rudas, L. Nádai, and K. Kósi, "Adaptive controllability of the Brusselator model with input coupling," In Proc of the $4^{\text {th }}$ IEEE Intl. Symp. on Logistics and Industrial Informatics (LINDI), Smolenice, Slovakia, pp. 157-162, 2012.

[13] B. Salimbahrami and B. Lohmann, Krylov Subspace Methods in Linear Model Order Reduction: Introduction and Invariance Properties. Scientific report, $2002 . \quad$ http://www.iat.unibremen.de/mitarbeiter/salimbahrami/ Invariance properties.pdf, 2002.

[14] Z. Bai, "Krylov subspace techniques for reduced-order modeling of large-scale dynamical systems," Applied Numerical Mathematics, vol. 43, pp. 9-44, 2002.

[15] C. Lanczos, "An iteration method for the solution of the eigenvalue problem of linear differential and integral operators," J. Res. Nat. Bureau Stan., vol. 45, pp. 255-282, 1950.

[16] W. Arnoldi, "The principle of minimized iterations in solution of the matrix eigenvalue problem," Quart. Appl. Math., vol. 9, pp. 17-29, 1951.

[17] H. Padé, Sur la répresentation approchée d'une fonction par des fractions rationelles (Thesis). Ann. École Nor. (3), 9, 1892, pp. 1-93 supplement, 1892.

[18] A. Bultheel and M. van Barvel, "Padé techniques for model reduction in linear system theory: A survey," J. Comput. Appl. Math., vol. 14, pp. 401-438, 1986.

[19] J. Liouville, "Mémoire sur le calcul des différentielles a indices quelconcues," J. Ecole Polytechn., vol. 3, pp. 71-162, 1832.

[20] A. Grünwald, "Über "begrenzte" Derivationen und deren Anwendung," Zeitshrift für angewandte Mathematik und Physik, vol. 12, pp. 41-48, 1867.

[21] J. Tenreiro Machado, "Fractional calculus and dynamical systems," Plenary lecture at the IEEE International Conference on Computational Cybernetics, 2006 (ICCC 2006), Tallinn, Estonia, 20-22 August, 2006, 2006.

[22] A. Gemant, "Method of analyzing experimental results obtained from elasto-viscous bodies," Physics, vol. 7, pp. 311-317, 1936.

[23] — " "On fractional differentials," The Phylosophical Magzine, vol. 25, pp. 540-549, 1938.

[24] A. Oustaloup, La Commande CRONE: Commande Robuste d'Ordre Non Entier. Hermes, 1991. 
[25] P. Lanusse, T. Poinot, O. Cois, A. Oustaloup, and J. Trigeassou, “Tuning of an active suspension system using a fractional controller and a closedloop tuning," Proc. of the 11th International Conference on Advanced Robotics (ICAR 2003), Coimbra, Portugal, June 30 - July 3, 2003, pp. 258-263, 2003.

[26] J. Tar, T. Haidegger, L. Kovács, K. Kósi, B. Botka, and I. Rudas, "Nonlinear order-reduced adaptive controller for a DC motor driven electric cart," In the Proc. of the $18^{\text {th }}$ International Conference on Intelligent Engineering Systems (INES 2014), Tihany, Hungary, July 3 5, 2014, pp. 73-78, 2014.

[27] J. Tar, J. Bitó, L. Nádai, and J. Tenreiro Machado, "Robust Fixed Point Transformations in adaptive control using local basin of attraction," Acta Polytechnica Hungarica, vol. 6, no. 1, pp. 21-37, 2009.

[28] A. Lyapunov, A general task about the stability of motion. (in Russian). $\mathrm{Ph} . \mathrm{D}$. Thesis, University of Kazan, Tatarstan (Russia), 1892.

[29] - Stability of motion. Academic Press, New-York and London, 1966

[30] J.-J. E. Slotine and W. Li, Applied Nonlinear Control. Prentice Hall International, Inc., Englewood Cliffs, New Jersey, 1991.

[31] R. Isermann, K. Lachmann, and D. Matko, Adaptive Control Systems. Prentice-Hall, New York DC, USA, 1992.

[32] C. Nguyen, S. Antrazi, Z.-L. Zhou, and C. Campbell Jr., "Adaptive control of a Stewart platform-based manipulator," Journal of Robotic Systems, vol. 10, no. 5, pp. 657-687, 1993.

[33] R. Kamnik, D. Matko, and T. Bajd, "Application of model reference adaptive control to industrial robot impedance control," Journal of Intelligent and Robotic Systems, vol. 22, pp. 153-163, 1998.

[34] K. Hosseini-Suny, H. Momeni, and F. Janabi-Sharifi, "Model Reference Adaptive Control design for a teleoperation system with output prediction," J Intell Robot Syst, vol. DOI 10.1007/s10846-010-9400-4, pp. 121, 2010.

[35] I. Sekaj and V. Veselý, "Robust output feedback controller design: Genetic Algorithm approach," IMA J Math Control Info, vol. 22, no. 3, pp. 257-265, 2005.

[36] J. Chen and W.-D. Chang, "Feedback linearization control of a two-link robot using a Multi-Crossover Genetic Algorithm," Expert Systems with Applications, vol. 2, no. 2 Part 2, pp. 4154-4159, 2009.

[37] P. Baranyi, L. Szeidl, P. Várlaki, and Y. Yam, "Definition of the HOSVDbased canonical form of polytopic dynamic models," In Proc. of the

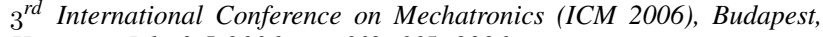
Hungary, July 3-5 2006, pp. 660-665, 2006.

[38] B. Takarics and P. Baranyi, "TP model-based robust stabilization of the 3 degrees-of-freedom aeroelastic wing section," Acta Polytechnica Hungarica, vol. 12, no. 1, pp. 209 - 228, 2014.

[39] G. Eigner, J. Tar, I. Rudas, and L. Kovács, "LPV-based quality interpretations on modeling and control of diabetes," Acta Polytechnica Hungarica, vol. 13, no. 1, pp. 171-190, 2016.

[40] T. J. Ypma, "Historical development of the Newton-Raphson method," SIAM Review, vol. 37, no. 4, pp. 531-551, 1995.

[41] J. Ortega and W. Rheinboldt, Iterative Solution of Nonlinear Equations in Several Variables. SIAM, 2000.

[42] P. Deuflhard, Newton Methods for Nonlinear Problems. Affine Invariance and Adaptive Algorithms, Springer Series in Computational Mathematics, Vol. 35. Springer, Berlin, 2004.

[43] S. Banach, "Sur les opérations dans les ensembles abstraits et leur application aux équations intégrales (About the Operations in the Abstract Sets and Their Application to Integral Equations)," Fund. Math., vol. 3, pp. 133-181, 1922.

[44] G. Eigner, J. Tar, and L. Kovács, "Adaptive control solution for T1DM control," Proc. of the 10th IEEE Intl. Sym. on Applied Computational Intelligence and Informatics (SACI 2015), May 21-23 2015, Timisoara, Romania, pp. 215-220, 2015.

[45] G. Eigner, P. Horváth, J. Tar, I. Rudas, and L. Kovács, "Application of Robust Fixed Point control in case of T1DM," In proc. of the IEEE International Conference on Systems, Man, and Cybernetics, October 10-13, 2015, Hong Kong (SMC 2015), pp. 2459-2463, 2015.

[46] J. Tar, K. Kósi, and T. Haidegger, "Generalized dynamic model of DC motors driven WMRs for RFPT-based order reduced adaptive control," 2nd Workshop on Design, Simulation, Optimization, and Control of Green Vehicles 22-23 September, 2014, Széchenyi István University, Gyór, Hungary, pp. 1-28, 2014.

[47] K. Kósi, T. Várkonyi, J. Tar, I. Rudas, and J. Bitó, "On the simulation of RFPT-based adaptive control of systems of 4th order response," In Proc. of the IEEE $11^{\text {th }}$ Intl. Symp. on Intelligent Systems and Informatics (SISY 2013), September 26-28, 2013, Subotica, Serbia, pp. 259-264, 2013.

[48] K. Kósi, J. Bitó, and J. Tar, "On the effects of strong asymmetries on the adaptive controllers based on robust fixed point transformations," In Proc. of the IEEE $10^{\text {th }}$ Jubilee Intl. Symp. on Intelligent Systems and Informatics (SISY 2012), Subotica, Serbia, pp. 259-264, 2012.

[49] K. Kósi, A. Dineva, and J. Tar, "Increased cycle time achieved by fractional derivatives in the adaptive control of the Brusselator model,' In Proc. of the $11^{\text {th }}$ IEEE Intl. Symp. on Applied Machine Intelligence and Informatics (SAMI), Herl'any, Slovakia, pp. 65-70, 2013.

[50] J. Tar, J. Bitó, and I. Rudas, "Contradiction resolution in the adaptive control of underactuated mechanical systems evading the framework of optimal controllers," Acta Polytechnica Hungarica, vol. 13, no. 1, pp. 97-121, 2016.

[51] J. Tar, J. Bitó, I. Rudas, and T. Várkonyi, "Decentralized adaptive control with fractional order elimination of obsolete information," In the Proc. of the 2011 Fourth International Conference on Emerging Trends in Engineering \& Technology, November 18 - 20, 2011, Mauritius, pp. 43-48, 2011.

[52] J. Tar, I. Rudas, A. Dineva, and A. Várkonyi-Kóczy, "Stabilization of a Modified Slotine-Li Adaptive Robot Controller by Robust Fixed Point Transformations," In Proc. of Recent Advances in Intelligent Control, Modelling and Simulation, 2014, Cambridge, MA, USA, pp. 35-40, 2014.

[53] A. Dineva, A. Várkonyi-Kóczy, and J. Tar, "Combination of RFPT-based adaptive control and classical model identification," In Proc. of the IEEE $12^{\text {th }}$ Intl. Symp. on Applied Machine Intelligence and Informatics (SAMI 2014), 2014, Herla'ny, Slovakia, pp. 35-40, 2014.

[54] C. De Souza, "Decentralized control of interconnected linear systems," Kybernetika, vol. 37, no. 3, pp. 309-323, 2001.

[55] J. Wu, T. Chen, and L. Wang, "Delay-dependent robust stability and h infinity control for jump linear systems with delays," In the Proc of the 2005 American Control Conference, June 8-10, 2005. Portland, OR, USA, vol. ThC02.2, pp. 2895-2900, 2005.

[56] S. Stojanovic, D. Debeljkovic, and I. Mladenovic, "Simple exponential stability criteria of linear discrete time-delay systems," SERBIAN JOURNAL OF ELECTRICAL ENGINEERING, vol. 5, no. 2, pp. 191-198, 2008.

[57] C. Briat, "Robust stability and stabilization of uncertain linear positive systems via integral linear constraints: L1- and linfinity-gains characterization," Cornell University Library, vol. http://arxiv.org/abs/1204.3554v3, no. arXiv:1204.3554 [cs.SY], pp. 1-26, 2012.

[58] E. Fridman and U. Shaked, "An improved stabilization method for linear time-delay systems," IEEE TRANSACTIONS ON AUTOMATIC CONTROL, vol. 47, no. 11, pp. 1931-1937, 2002.

[59] E. Fridman, "A new lyapunov technique for robust control of systems with uncertain non-small delays," IMA Journal of Mathematical Control and Information, vol. doi:10.1093/imamci/dni051, pp. 1-15, 2005.

[60] A. Tchangani, M. Dambrine, and J. Richard, "Delay-dependent criteria for robust stability of time-varying delay systems," In the Proc. of the 37th IEEE Conference on Decision \& Control, Tampa, Florida, USA, December 1998, pp. 4051-4056, 1998.

[61] V. Kolmanovskii, S.-I. Niculescu, and D. Richard, "On the lyapunovkrasovskii functionals for stability analysis of linear delay systems," International Journal of Control, vol. 72, no. 4, pp. 374-384.

[62] M. Wu, Y. He, J.-H. She, and G.-P. Liu, "Delay-dependent criteria for robust stability of time-varying delay systems," Automatica, vol. 40, no. 8, pp. 1435-1439, 2004.

[63] M. Belhaouane, M. Ghariani, H. Ayadi, and N. Braiek, "Improved results on robust stability analysis and stabilization for a class of uncertain nonlinear systems," Mathematical Problems in Engineering, vol. 2010, no. doi:10.1155/2010/724563, pp. 1-24, 2010.

[64] V. Utkin, Sliding Modes in Optimization and Control Problems. Springer Verlag, New York, 1992.

[65] K. Széll and P. Korondi, "Mathematical basis of sliding mode control of an uninterruptible power supply," Acta Polytechnica Hungarica, vol. 11, no. 03 , pp. $87-106,2014$.

[66] A. Mechter, K. Kemih, and M. Ghanes, "Sliding mode control of a wind turbine with exponential reaching law," Acta Polytechnica Hungarica, vol. 12 , no. 03 , pp. $167-183,2015$.

[67] K. Kósi, S. Hajdu, J. Bitó, and J. Tar, "Chaos formation and reduction in Robust Fixed Point Transformations based adaptive control," In Proc. of the 4th IEEE Intl. Conf. on Nonlinear Science and Complexity (NSC 2012), Budapest, Hungary, pp. 211-216, 2012. 